\title{
Invariant manifolds, global attractors and almost periodic solutions of nonautonomous difference equations
}

\author{
David Cheban $^{\mathrm{a}, *}$, Cristiana Mammana ${ }^{\mathrm{b}}$ \\ ${ }^{a}$ Department of Mathematics and Informatics, State University of Moldova, \\ A. Mateevich Street 60, MD-2009 Chişinău, Moldova \\ ${ }^{\mathrm{b}}$ Institute of Economics and Finances, University of Macerata, str. Crescimbeni 20, \\ I-62100 Macerata, Italy
}

\begin{abstract}
The article is devoted to the study of quasi-linear nonautonomous difference equations: invariant manifolds, compact global attractors, almost periodic and recurrent solutions and chaotic sets. First, we prove that such equations admit an invariant continuous section (an invariant manifold). Then, we obtain the conditions for the existence of a compact global attractor and characterize its structure. Third, we derive a criterion for the existence of almost periodic and recurrent solutions of the quasi-linear nonautonomous difference equations. Finally, we prove that quasi-linear maps with chaotic base admit a chaotic compact invariant set. The obtained results are applied while studying triangular maps: invariant manifolds, compact global attractors, almost periodic and recurrent solutions and chaotic sets.
\end{abstract}

(C) 2003 Elsevier Ltd. All rights reserved.

MSC: primary: 34C35; 34D20; 34D40; 34D45; 58F10; 58F12; 58F39; secondary: 35B $35 ; 35 \mathrm{~B} 40$

Keywords: Chaos; Triangular maps; Nonautonomous dynamical systems with discrete time; Skew-product flow; Global attractor; Almost periodic and recurrent solutions

\section{Introduction}

In the qualitative theory of differential and difference equations nonlocal problems play the important role (See, for instance, [1-16] and the bibliography therein). It

\footnotetext{
* Corresponding author.

E-mail addresses: cheban@usm.md (D. Cheban), cmamman@tin.it (C. Mammana).
} 
refers to questions of boundedness, periodicity, almost periodicity, stability by Poisson, asymptotic behaviour, dissipativity etc.

The present work belongs to this direction and is dedicated to the study of quasi-linear nonautonomous difference equations: invariant manifolds, compact global attractors, almost periodic and recurrent solutions, invariant manifolds and chaotic sets. We prove that such equations admit an invariant continuous section (an invariant manifold). Then, we obtain the conditions of the existence of a compact global attractor and characterize its structure. We give a criterion for the existence of almost periodic and recurrent solutions of the quasi-linear nonautonomous difference equations. Finally, we prove that quasi-linear difference equations with chaotic base admits a chaotic compact invariant set.

The obtained results are applied while studying triangular maps: invariant manifolds, compact global attractors, almost periodic and recurrent solutions and chaotic sets. Triangular maps (see, for example, $[7,10,11]$ ) are close to one-dimensional maps in the sense that some important dynamical features extend to triangular maps. On the other hand, they already display other important properties which are typical for higher dimensional maps and cannot be found in the one-dimensional maps.

Below, we give a new approach concerning the study of triangular maps. We study this problem in the framework of nonautonomous dynamical systems (cocycles) with discrete time. In our work the main tool in the study of triangular maps are continuous invariant sections (selectors) of cocycle.

This paper is organized as follows.

In Section 2, we establish the relation between triangular maps and nonautonomous dynamical systems with discrete time.

In Section 3, we study linear nonautonomous dynamical systems with discrete time and prove that they admit a unique continuous invariant section-invariant manifold (Theorem 3.7).

Section 4 is devoted to the study of the existence of invariant sections of quasi-linear nonautonomous dynamical systems with discrete time (Theorems 4.1 and 4.2).

In Section 5, we prove the existence of compact global attractors of quasi-linear dynamical systems (Theorems 5.2 and 5.6) and give the description of the structure of these attractors (Theorems 5.9 and 5.11).

Section 6 is devoted to the study of almost periodic and recurrent solutions of quasi-linear difference equations (Theorems 6.10 and 6.11).

In Section 7, we introduce the notion of pseudo-recurrent solution and prove that quasi-linear dynamical systems with pseudo-recurrent base admit a pseudo-recurrent solution (Theorem 7.3).

Section 8 is devoted to the study of chaos in triangular maps and nonautonomous dynamical systems with discrete time (Theorem 8.7).

\section{Triangular maps and nonautonomous dynamical systems}

Let $W$ and $\Omega$ be two complete metric spaces and denote by $X:=W \times \Omega$ its Cartesian product. Recall (see, for example, $[10,11,14]$ ) that a continuous map $F: X \rightarrow X$ is 
called triangular, if there are two continuous maps $f: W \times \Omega \rightarrow W$ and $g: \Omega \rightarrow \Omega$ such that $F=(f, g)$, i.e. $F(x)=F(u, \omega)=(f(u, \omega), g(\omega))$ for all $x=:(u, \omega) \in X$.

Consider a system of difference equations

$$
\left\{\begin{array}{l}
u_{n+1}=f\left(u_{n}, \omega_{n}\right), \\
\omega_{n+1}=g\left(\omega_{n}\right)
\end{array}\right.
$$

for all $n \in \mathbb{Z}_{+}$, where $\mathbb{Z}_{+}$is the set of all nonnegative integer numbers.

Along with system (1) we consider the family of equations

$$
u_{n+1}=f\left(u_{n}, g^{n} \omega\right) \quad(\omega \in \Omega)
$$

which is equivalent to system (1). Let $\varphi(n, u, \omega)$ be a solution of Eq. (2) passing through the point $u \in W$ for $n=0$. It is easy to verify that the map $\varphi: \mathbb{Z}_{+} \times W \times \Omega \rightarrow$ $W((n, u, \omega) \mapsto \varphi(n, u, \omega))$ satisfies the following conditions:

(i) $\varphi(0, u, \omega)=u$ for all $u \in W$ and $\omega \in \Omega$;

(ii) $\varphi(n+m, u, \omega)=\varphi(n, \varphi(m, u, \omega), \sigma(m, \omega))$ for all $n, m \in \mathbb{Z}_{+}, u \in W$ and $\omega \in \Omega$, where $\sigma(n, \omega):=g^{n} \omega$

(iii) the map $\varphi: \mathbb{Z}_{+} \times W \times \Omega \rightarrow W$ is continuous.

Denote by $\left(\Omega, \mathbb{Z}_{+}, \sigma\right)$ the semi-group dynamical system generated by positive powers of the map $g: \Omega \rightarrow \Omega$, i.e. $\sigma(n, \omega):=g^{n} \omega$ for all $n \in \mathbb{Z}_{+}$and $\omega \in \Omega$.

Definition 2.1. Recall $[2,3,13]$ that a triple $\left\langle W, \varphi,\left(\Omega, \mathbb{Z}_{+}, \sigma\right)\right\rangle$ (or briefly $\varphi$ ) is called a cocycle (or nonautonomous dynamical system) over the dynamical system $\left(\Omega, \mathbb{Z}_{+}, \sigma\right)$ with fiber $W$.

Let $X:=W$ and $\left(X, \mathbb{Z}_{+}, \pi\right)$ be a dynamical system on $X$, where $\pi(n,(u, \omega)):=$ $(\varphi(n, u, \omega), \sigma(n, \omega))$ for all $u \in W$ and $\omega \in \Omega$, then $\left(X, \mathbb{Z}_{+}, \pi\right)$ is called [13] a skewproduct dynamical system, generated by the cocycle $\left\langle W, \varphi,\left(\Omega, \mathbb{Z}_{+}, \sigma\right)\right\rangle$.

Remark 2.2. Thus, the reasoning above shows that every triangular map generates a cocycle and, obviously, vice versa, i.e. having a cocycle $\left\langle W, \varphi,\left(\Omega, \mathbb{Z}_{+}, \sigma\right)\right\rangle$ we can define a triangular map $F: W \times \Omega \rightarrow W \times \Omega$ by the equality

$$
F(u, \omega):=(f(u, \omega), g(\omega)),
$$

where $f(u, \omega):=\varphi(1, u, \omega)$ and $g(\omega):=\sigma(1, \omega)$ for all $u \in W$ and $\omega \in \Omega$. The dynamical system defined by the positive powers of map $F: X \rightarrow X(X:=W \times \Omega)$ coincides with the skew-product dynamical system, generated by cocycle $\left\langle W, \varphi,\left(\Omega, \mathbb{Z}_{+}, \sigma\right)\right\rangle$.

Taking into consideration this remark we can study triangular maps in the framework of nonautonomous dynamical systems (cocycles) with discrete time.

Definition 2.3. A map $\gamma: \mathbb{Z} \rightarrow \Omega$ (respectively, $\alpha: \mathbb{Z} \rightarrow X$, where $X:=W \times \Omega$ ) is called an entire trajectory of the dynamical system $\left(\Omega, \mathbb{Z}_{+}, \sigma\right)$ (respectively, of the skew-product dynamical system $\left(X, \mathbb{Z}_{+}, \pi\right)$, where $\pi:=(\varphi, \sigma)$ and $\left\langle W, \varphi,\left(\Omega, \mathbb{Z}_{+}, \sigma\right)\right\rangle$ is 
a cocycle over $\left(\Omega, \mathbb{Z}_{+}, \sigma\right)$ with fiber $W$ ) passing through the point $\omega \in \Omega$ (respectively, $x:=(u, \omega) \in X)$, if $\gamma(0)=\omega(\operatorname{resp} . \alpha(0)=x)$ and $\gamma(n+m)=\sigma(m, \gamma(n))(\operatorname{resp} . \alpha(n+m)$ $=\pi(m, \alpha(n))$ for all $n \in \mathbb{Z}$ and $m \in \mathbb{Z}_{+}$.

Denote by $\Phi_{\omega}$ the set of all the entire trajectories of the semi-group dynamical system $\left(\Omega, \mathbb{Z}_{+}, \sigma\right)$ passing through the point $\omega \in \Omega$ at the initial moment $n=0$ and $\Phi:=\bigcup\left\{\Phi_{\omega} \mid \omega \in \Omega\right\}$.

Definition 2.4. A map $v: \mathbb{Z} \rightarrow W$ is called an entire trajectory of the cocycle $\langle W, \varphi$, $\left.\left(\Omega, \mathbb{Z}_{+}, \sigma\right)\right\rangle$ passing through the point $u \in W$ if there are $\omega \in \Omega$ and $\gamma_{\omega} \in \Phi_{\omega}$ such that the map $\alpha: \mathbb{Z} \rightarrow X(X:=W \times \Omega)$ defined by the equality $\alpha:=\left(v, \gamma_{\omega}\right)$ is an entire trajectory of the skew-product dynamical system $\left(X, \mathbb{Z}_{+}, \pi\right)$, i.e. $\alpha \in \Phi_{x}$ and $x:=(u, \omega)$.

\section{Linear nonautonomous dynamical systems}

Let $\Omega$ be a compact metric space and $\left(\Omega, \mathbb{Z}_{+}, \sigma\right)$ be a semi-group dynamical system on $\Omega$ with discrete time.

Definition 3.1. Recall that a subset $A \subseteq \Omega$ is called invariant (positively invariant, negatively invariant) if $\sigma^{n} A=A\left(\sigma^{n} A \subseteq A, A \subseteq \sigma^{n} A\right)$ for all $n \in \mathbb{Z}_{+}$, where $\sigma^{n}:=$ $\sigma(n, \cdot): \Omega \rightarrow \Omega$.

Below we will suppose that the set $\Omega$ is invariant, i.e. $\sigma^{n} \Omega=\Omega$ for all $n \in \mathbb{Z}_{+}$. Let $E$ be a finite-dimensional Banach space with the norm $|\cdot|$ and $W$ be a complete metric space. Denote by $L(E)$ the space of all linear continuous operators on $E$ and by $C(\Omega, W)$ the space of all the continuous functions $f: \Omega \rightarrow W$ endowed with the compact-open topology, i.e. the uniform convergence on compact subsets in $\Omega$. The results of this section will be used in the next sections.

Consider a linear equation

$$
u_{n+1}=A\left(\sigma^{n} \omega\right) u_{n} \quad\left(\omega \in \Omega, \sigma^{n} \omega:=\sigma(n, \omega)\right)
$$

and an inhomogeneous equation

$$
u_{n+1}=A\left(\sigma^{n} \omega\right) u_{n}+f\left(\sigma^{n} \omega\right),
$$

where $A \in C(\Omega, L(E))$ and $f \in C(\Omega, E)$.

Definition 3.2. Recall that a linear bounded operator $P: E \rightarrow E$ is called a projection, if $P^{2}=P$, where $P^{2}:=P \circ P$.

Definition 3.3. Let $U(n, \omega)$ be the operator of Cauchy (a solution operator) of linear Eq. (3). Following [5] we will say that Eq. (3) has an exponential dichotomy on $\Omega$, if there exists a continuous projection valued function $P: \Omega \rightarrow L(E)$ satisfying:

(i) $P\left(\sigma^{n} \omega\right) U(n, \omega)=U(n, \omega) P(\omega)$;

(ii) $U_{Q}(n, \omega)$ is invertible as an operator from $\operatorname{Im} Q(\omega)$ to $\operatorname{Im} Q\left(\sigma^{n} \omega\right)$, where $U_{Q}(n, \omega)$ $:=U(n, \omega) Q(\omega)$ 
(iii) there exist constants $0<v<1$ and $N>0$ such that

$$
\left\|U_{P}(n, \omega)\right\| \leqslant N q^{n} \quad \text { and } \quad\left\|U_{Q}(n, \omega)^{-1}\right\| \leqslant N q^{n}
$$

for all $\omega \in \Omega$ and $n \in \mathbb{Z}_{+}$, where $U_{P}(n, \omega):=U(n, \omega) P(\omega)$.

Let $\omega \in \Omega$ and $\gamma_{\omega} \in \Phi_{\omega}$. Consider a difference equation

$$
u_{n+1}=A\left(\gamma_{\omega}(n)\right) u_{n}+f\left(\gamma_{\omega}(n)\right),
$$

and the corresponding homogeneous linear equation

$$
u_{n+1}=A\left(\gamma_{\omega}(n)\right) u_{n} \quad(\omega \in \Omega) .
$$

Let $(X, \rho)$ be a metric space with distance $\rho$. Denote by $C(\mathbb{Z}, X)$ the space of all the functions $f: \mathbb{Z} \rightarrow X$ equipped with a pointwise topology. This topology can be metricised. For example, by the equality

$$
d\left(f_{1}, f_{2}\right):=\sum_{1}^{+\infty} \frac{1}{2^{n}} \frac{d_{n}\left(f_{1}, d_{2}\right)}{1+d_{n}\left(f_{1}, d_{2}\right)},
$$

where $d_{n}\left(f_{1}, d_{2}\right):=\max \left\{\rho\left(f_{1}(k), f_{2}(k)\right) \mid k \in[-n, n]\right\}$, a distance is defined on $C(\mathbb{Z}, X)$ which generates the pointwise topology.

If $x \in X$ and $A, B \subseteq X$, then denote by $\rho(x, A):=\inf \{\rho(x, a) \mid a \in A\}$ and $\beta(A, B):=$ $\sup \{\rho(a, B) \mid a \in A\}$ the semi-distance of Hausdorff.

Theorem 3.4. Suppose that linear equation (3) has an exponential dichotomy on $\Omega$. Then for $f \in C(\Omega, E)$ the following statements hold:

(i) the set $I_{\omega}:=\left\{u \in E \mid \exists \gamma_{\omega} \in \Phi_{\omega}\right.$ such that Eq. (5) admits a bounded solution $\psi_{\omega}$ defined on $\mathbb{Z}$ with the initial condition $\left.\psi_{\omega}(0)=u\right\}$ is nonempty and compact;

(ii) $\varphi\left(n, I_{\omega}, \omega\right)=I_{\sigma(n, \omega)}$ for all $n \in \mathbb{Z}_{+}$and $\omega \in \Omega$, where $\varphi(n, u, \omega)$ is a solution of Eq. (4) with the condition $\varphi(0, u, \omega)=u$ and $\varphi(n, M, \omega):=\{\varphi(n, u, \omega) \mid u \in M\}$;

(iii) the map $\omega \rightarrow I_{\omega}$ is upper-semi-continuous, i.e.

$$
\lim _{\omega \rightarrow \omega_{0}} \beta\left(I_{\omega}, I_{\omega_{0}}\right)=0
$$

for every $\omega_{0} \in \Omega$, where $\beta$ is the semi-distance of Hausdorff;

(iv) the set $I:=\bigcup\left\{I_{\omega} \mid \omega \in \Omega\right\}$ is compact.

Proof. Let $\omega \in \Omega$. Since $\Omega$ is compact and invariant, the set $\Phi_{\omega} \neq \emptyset$. We fix $\gamma_{\omega} \in \Phi_{\omega}$. Under the conditions of Theorem 3.4, Eq. (6) has an exponential dichotomy on $\Omega$ with the same constants $N$ and $q$ that in Eq. (3). Then Eq. (5) admits the unique solution $v_{\omega}: \mathbb{Z} \rightarrow E$ with the condition

$$
\left\|v_{\omega}\right\|_{\infty} \leqslant N \frac{1+q}{1-q}\|f\|_{\infty} \leqslant N \frac{1+q}{1-q}\|f\|
$$

where $\|f\|:=\sup \{|f(\omega)| \mid \omega \in \Omega\}$ and $\left\|v_{\omega}\right\|_{\infty}:=\sup \left\{\left|v_{\omega}(n)\right| \mid n \in \mathbb{Z}\right\} \quad$ (see, for example, $[6,9])$. Thus, the set $I_{\omega}$ is not empty. From the continuity of the function 
$\varphi: \mathbb{Z}_{+} \times E \times \Omega \rightarrow E$ and inequality (7) follows that the set $I_{\omega}$ is closed, bounded and

$$
|u| \leqslant N \frac{1+q}{1-q}\|f\|
$$

for all $u \in I_{\omega}$ and $\omega \in \Omega$.

The second statement of the theorem follows from the equality $S_{h}\left(\Phi_{\omega}\right)=\Phi_{\sigma(h, \omega)}$ $(h \in \mathbb{Z})$, where $S_{h} \gamma_{\omega}$ is an $h$-translation of the trajectory $\gamma_{\omega}$, i.e. $S_{h} \gamma_{\omega}(n):=\gamma_{\omega}(n+h)$ for all $n \in \mathbb{Z}$.

We will prove now the third statement. Let $\omega_{0} \in \Omega, \omega_{k} \rightarrow \omega_{0}, u_{k} \in I_{\omega_{k}}$ and $u_{k} \rightarrow u$. To prove our statement it is sufficient to show that $u \in I_{\omega_{0}}$. Since $u_{k} \in I_{\omega_{k}}$, there is a trajectory $\gamma_{\omega_{k}} \in \Phi_{\omega_{k}}$ such that $\gamma_{\omega_{k}}$ converges to $\gamma_{\omega_{0}} \in \Phi_{\omega_{0}}$ in $C(\mathbb{Z}, \Omega)$ and the equation

$$
u_{n+1}=A\left(\gamma_{\omega_{k}}(n)\right) u_{n}+f\left(\gamma_{\omega_{k}}(n)\right)
$$

has a solution $v_{\omega_{k}}$ with the initial condition $v_{\omega_{k}}(0)=u_{k}$ and satisfying inequality (7), i.e.

$$
\left|v_{\omega_{k}}(n)\right| \leqslant N \frac{1+q}{1-q}\|f\|_{\infty} \leqslant N \frac{1+q}{1-q}\|f\|
$$

for all $n \in \mathbb{Z}$ and $k \in \mathbb{N}$. It is clear that the sequence $\left\{v_{\omega_{k}(n)}\right\}$ converges for every $n \in \mathbb{Z}$. By Tihonoff's theorem the sequence $\left\{v_{\omega_{k}}\right\} \subset C(\mathbb{Z}, E)$ is relatively compact. From equality (8) and inequality (9) follows that every limit point of the sequence $\left\{v_{\omega_{k}}\right\}$ is a bounded on $\mathbb{Z}$ solution of the equation

$$
u_{n+1}=A\left(\gamma_{\omega_{0}}(n)\right) u_{n}+f\left(\gamma_{\omega_{0}}(n)\right)
$$

Taking into account that Eq. (10) admits a unique bounded on $\mathbb{Z}$ solution, we obtain the convergence of the sequence $\left\{v_{\omega_{k}}\right\}$ in the space $C(\mathbb{Z}, E)$. We put $v_{0}:=\lim _{k \rightarrow+\infty} v_{\omega_{k}}$. It is easy to see that $v_{0}(0)=u$ and, consequently, $u \in I_{\omega_{0}}$.

To prove the fourth assertion it is sufficient to remark that for every $\omega \in \Omega$ the set $I_{\omega}$ is compact, the map $F: \omega \rightarrow I_{\omega}\left(F(\omega):=I_{\omega}\right)$ is upper-semicontinuous and, consequently, the set $I:=\bigcup\left\{I_{\omega} \mid \omega \in \Omega\right\}=F(\Omega)$ is compact. The theorem is completely proved.

Lemma 3.5. Let $\omega \in \Omega$ and $\gamma_{\omega}^{l} \in \Phi_{\omega}(l=1,2)$. Under the conditions of Theorem 3.4 we have

$$
\left|v_{\omega}^{1}(n)-v_{\omega}^{2}(n)\right| \leqslant N q^{n}\left|u^{1}-u^{2}\right|
$$

for all $n \in \mathbb{N}$, where $v_{\omega}^{l}$ is a bounded on $\mathbb{Z}$ solution of the equation

$$
u_{n+1}=A\left(\gamma_{\omega}^{l}(n)\right) u_{n}+f\left(\gamma_{\omega}^{l}(n)\right)
$$

$(l=1,2)$ and $u_{i}=v_{\omega}^{i}(0)(i=1,2)$.

Proof. Denote $v(n):=v_{\omega}^{1}(n)-v_{\omega}^{2}(n)$, then the sequence $v$ is a bounded on $\mathbb{Z}_{+}$solution of Eq. (6), because $\gamma_{\omega}^{1}(n)=\gamma_{\omega}^{2}(n)=\sigma(n, \omega)$ for all $n \in \mathbb{Z}_{+}$. Since Eq. (3) (and, consequently, Eq. (6) too) has an exponential dichotomy, we have $|v(n)| \leqslant N q^{n}|v(0)|$ 
for all $n \in \mathbb{Z}_{+}$. Taking into consideration that $v(0)=u^{1}-u^{2}$, we obtain the required statement. The lemma is proved.

Lemma 3.6. Under the conditions of Theorem 3.4 for every $\omega \in \Omega$ the set $I_{\omega}$ contains a single point $u_{\omega}$.

Proof. Suppose that the statement of the lemma is not true. Then there exists $\omega_{0} \in \Omega$ such that $I_{\omega_{0}}$ contains at least two points $u_{1}, u_{2}\left(u_{1} \neq u_{2}\right)$. Let $v_{i}(i=1,2)$ be a bounded on $\mathbb{Z}$ solution of Eq. (10) with the condition $v_{\omega}^{i}(0)=u_{i}(i=1,2)$. According to inequality (11), we have

$$
\left|v_{\omega}^{1}(-n)-v_{\omega}^{2}(-n)\right| \geqslant N q^{-n}\left|u^{1}-u^{2}\right|
$$

for all $n \in \mathbb{N}$. On the other hand, since $v_{\omega}^{l}(l=1,2)$ is a bounded on $\mathbb{Z}$ solution of Eq. (12), we have

$$
\sup _{n \in \mathbb{Z}}\left|v_{\omega}^{1}(n)-v_{\omega}^{2}(n)\right|<+\infty .
$$

Inequalities (13) and (14) are contradictory. The contradiction obtained proves our affirmation. The lemma is proved.

Theorem 3.7. Under the condition of Theorem 3.4 there exists a unique continuous function $v: \Omega \rightarrow E$ satisfying the following conditions:

(a) the equality

$$
v(\sigma(n, \omega))=\varphi(n, v(\omega), \omega)
$$

holds for all $n \in \mathbb{Z}_{+}$and $\omega \in \Omega$, where $\varphi(n, u, \omega)$ is the unique solution of Eq. (4) with the initial condition $\varphi(0, u, \omega)=u$.

(b)

$$
\|v\| \leqslant N \frac{1+q}{1-q}\|f\|
$$

Proof. This affirmation follows directly from Theorem 3.4 and Lemmas 3.5 and 3.6.

Definition 3.8. A dynamical system $\left(\Omega, \mathbb{Z}_{+}, \sigma\right)$ is said to be invertible, if the map $f:=\sigma(1, \cdot): \Omega \rightarrow \Omega$ is invertible and, consequently, on $\Omega$ there is defined a group dynamical system $(\Omega, \mathbb{Z}, \sigma)$, where $\pi(-n, \cdot):=\left(\pi(n, \cdot)^{-1}\right.$.

Remark 3.9. In case the dynamical system $\left(\Omega, \mathbb{Z}_{+}, \sigma\right)$ is invertible, Theorem 3.7 is well known (see, for example, [5]).

\section{Quasi-linear nonautonomous dynamical systems}

Let us consider the following quasi-linear equation

$$
u_{n+1}=A\left(\sigma^{n} \omega\right) u_{n}+f\left(\sigma^{n} \omega\right)+F\left(u_{n}, \sigma^{n} \omega\right),
$$

where $A \in C(\Omega, L(E)), f \in C(\Omega, E)$ and $F \in C(E \times \Omega, E)$. 
Theorem 4.1. Assume that there exist positive numbers $L<L_{0}:=(1-q) /(N(1+q))$ and $r<r_{0}:=\varepsilon_{0}(N(1+q)) /(1-q)\left(1-N L_{0}(1+q) /(1-q)\right)^{-1}$ such that

$$
\left\|F\left(x_{1}, \omega\right)-F\left(x_{2}, \omega\right)\right\| \leqslant L\left\|x_{1}-x_{2}\right\|
$$

for all $\omega \in \Omega$ and $x_{1}, x_{2} \in B[Q, r]=\{x \in E \mid \rho(x, Q) \leqslant r\}$, where $Q:=v(\Omega), v \in C(\Omega, E)$ is the unique function from $C(\Omega, E)$ arising in Theorem 3.7 and $\varepsilon_{0}=\max _{\omega \in \Omega} \| F(v(\omega)$, $\omega) \|$. Then there exists a function $w \in C(\Omega, B[Q, r])$ such that

$$
w\left(\sigma^{n} \omega\right)=\psi(n, w(\omega), \omega)
$$

for all $n \in \mathbb{Z}_{+}$and $\omega \in \Omega$, where $\psi(\cdot, u, \omega)$ is the unique solution of quasi-linear Eq. (15) with the initial condition $\psi(0, u, \omega)=u$.

Proof. Let $u:=v+v\left(\sigma^{n} \omega\right)$. Then from Eq. (15) we get

$$
v_{n+1}=A\left(\sigma^{n} \omega\right) v_{n}+F\left(v+v\left(\sigma^{n} \omega\right), \sigma^{n} \omega\right) .
$$

Let $0<r<r_{0}$ and $\alpha \in C(\Omega, B[Q, r])$, where $B[Q, r]:=\{u \in E \mid \rho(u, Q) \leqslant r\}$. Consider the equation

$$
v_{n+1}=A\left(\sigma^{n} \omega\right) v_{n}+\tilde{f}\left(\sigma^{n} \omega\right)
$$

where $\tilde{f}(\omega):=F(\alpha(\omega)+v(\omega), \omega)$ for all $\omega \in \Omega$. According to Theorem 3.7 , there exists a unique function $v_{\alpha} \in C(\Omega, E)$ with properties (a) and (b). In virtue of Theorem 3.7 , we have

$$
\begin{aligned}
\left\|v_{\alpha}\right\| \leqslant & N \frac{1+q}{1-q} \max _{\omega \in \Omega}\|F(\alpha(\omega)+v(\omega), \omega)\| \\
\leqslant & N \frac{1+q}{1-q} \max _{\omega \in \Omega}|F(\alpha(\omega)+v(\omega), \omega)-F(\gamma(\omega), \omega)| \\
& +N \frac{1+q}{1-q} \max _{\omega \in \Omega}|F(\gamma(\omega), \omega)| \\
\leqslant & N \frac{1+q}{1-q} L\|\alpha\|+N \frac{1+q}{1-q} \varepsilon_{0} \leqslant N \frac{1+q}{1-q} L r+\frac{2 N}{v} \varepsilon_{0} \\
\leqslant & N \frac{1+q}{1-q} L_{0} r_{0}+N \frac{1+q}{1-q} \varepsilon_{0}=r_{0}
\end{aligned}
$$

and, consequently, $\mathscr{F}\left(C\left(\Omega, B\left[Q, r_{0}\right]\right)\right) \subseteq C\left(\Omega, B\left[Q, r_{0}\right]\right)$, where $\left.\mathscr{F}: C(\Omega, B[Q, r])\right) \rightarrow$ $C(\Omega, E)$ is the map defined by the equality $\mathscr{F}(\alpha):=v_{\alpha}$.

Now, we will show that the map $\mathscr{F}: C\left(\Omega, B\left[Q, r_{0}\right]\right) \rightarrow C\left(\Omega, B\left[Q, r_{0}\right]\right)$ is a Lipschitzian one. In fact, according to Theorem 3.7 we have

$$
\begin{aligned}
\left\|\mathscr{F}\left(\alpha_{1}\right)-\mathscr{F}\left(\alpha_{2}\right)\right\| & \leqslant N \frac{1+q}{1-q} \max _{\omega \in \Omega}\left|F\left(\alpha_{1}(\omega)+v(\omega), \omega\right)-F\left(\alpha_{2}(\omega)+v(\omega), \omega\right)\right| \\
& \leqslant N \frac{1+q}{1-q} L \max _{\omega \in \Omega}\left|\alpha_{1}(\omega)-\alpha_{2}(\omega)\right| .
\end{aligned}
$$


We note that $N[(1+q) /(1-q)] L \leqslant N[(1+q) /(1-q)] L_{0}<1$. Thus, the map $\mathscr{F}$ is a contraction and, consequently, by Banach fixed point theorem, there exists a unique function $\alpha \in C\left(\Omega, B\left[Q, r_{0}\right]\right)$ such that $\mathscr{F}(\alpha)=\alpha$. To finish the proof of the theorem it is sufficient to put $w:=\gamma+\alpha$.

We now consider a perturbed quasi-linear equation

$$
u_{n+1}=A\left(\sigma^{n} \omega\right) u_{n}+f\left(\sigma^{n} \omega\right)+\lambda F\left(u_{n}, \sigma^{n} \omega\right) \text {, }
$$

where $\lambda \in\left[-\lambda_{0}, \lambda_{0}\right]\left(\lambda_{0}>0\right)$ is a small parameter.

Theorem 4.2. Assume that there exist positive numbers $r$ and $L$ such that

$$
\left|F\left(u_{1}, \omega\right)-F\left(u_{2}, \omega\right)\right| \leqslant L\left|u_{1}-u_{2}\right|
$$

for all $\omega \in \Omega$ and $u_{1}, u_{2} \in B[Q, r]$. Then for $\lambda$ small enough there exists a unique function $v_{\lambda} \in C(\Omega, B[Q, r])$ such that

$$
v_{\lambda}\left(\sigma^{n} \omega\right)=\psi_{\lambda}\left(n, v_{\lambda}(\omega), \omega\right)
$$

for all $n \in \mathbb{Z}_{+}$and $\omega \in \Omega$, where $\psi_{\lambda}(\cdot, u, \omega)$ is the unique solution of Eq. (16) with the initial condition $\psi_{\lambda}(0, u, \omega)=u$. Moreover,

$$
\lim _{\lambda \rightarrow 0} \max _{\omega \in \Omega}\left|v_{\lambda}(\omega)-v(\omega)\right|=0
$$

where $v \in C(\omega, E)$ is defined in Theorem 3.7.

Proof. We can prove the existence of $v_{\lambda}$ by a slight modification of the proof of Theorem 4.1.

To prove (17) we note that

$$
\left|F\left(v_{\lambda}(\omega), \omega\right)\right| \leqslant\left|F\left(v_{\lambda}(\omega), \omega\right)-F(v(\omega), \omega)\right|+|F(v(\omega), \omega)| \leqslant L r+\varepsilon_{0} .
$$

Denote $\Delta_{\lambda}(\omega):=v_{\lambda}(\omega)-v(\omega)(\omega \in \Omega)$. It is easy to see that the sequence $\left\{\Delta_{\lambda}\left(\sigma^{n} \omega\right)\right\}_{n \in \mathbb{Z}}$ is the unique bounded on $\mathbb{Z}$ solution of the equation

$$
z_{n+1}=A\left(\sigma^{n} \omega\right) z_{n}+\lambda F\left(v_{\lambda}\left(\sigma^{n}(\omega), \sigma^{n} \omega\right)\right)
$$

and, consequently,

$$
\begin{aligned}
\left|v_{\lambda}\left(\sigma^{n} \omega\right)-v\left(\sigma^{n} \omega\right)\right| & =\left|\Delta\left(\sigma^{n} \omega\right)\right| N \frac{1+q}{1-q} \sup _{n \in \mathbb{Z}}\left|\lambda F\left(v_{\lambda}\left(\sigma^{n} \omega\right), \sigma^{n} \omega\right)\right| \\
& \leqslant|\lambda| N \frac{1+q}{1-q}\left(L r+\varepsilon_{0}\right)
\end{aligned}
$$


for all $\omega \in \Omega, n \in \mathbb{Z}$ and $\lambda \in\left[-\lambda_{0}, \lambda_{0}\right]$. Thus, from inequality (18) we obtain

$$
\left|v_{\lambda}(\omega)-v(\omega)\right| \leqslant|\lambda| N \frac{1+q}{1-q}\left(L r+\varepsilon_{0}\right)
$$

for all $\omega \in \Omega$ and $\lambda \in\left[-\lambda_{0}, \lambda_{0}\right]$. Passing to limit in inequality (19) as $\lambda \rightarrow 0$, we obtain (17).

\section{Global attractors of quasi-linear triangular systems}

Consider a difference equation

$$
u_{n+1}=f\left(u_{n}, \sigma^{n} \omega\right) \quad(\omega \in \Omega) .
$$

Denote by $\varphi(n, u, \omega)$ a unique solution of Eq. (20) with the initial condition $\varphi(0, u, \omega)=u$.

Definition 5.1. Eq. (20) is said to be dissipative, if there exists a positive number $r$ such that

$$
\limsup _{n \rightarrow+\infty}|\varphi(n, u, \omega)| \leqslant r
$$

for all $u \in E$ and $\omega \in \Omega$.

Consider a quasi-linear equation

$$
u_{n+1}=A\left(\sigma^{n} \omega\right) u_{k}+F\left(u_{k}, \sigma^{n} \omega\right),
$$

where $A \in C(\Omega,[E])$ and the function $F \in C(E \times \Omega, E)$ satisfies to "the condition of smallness".

Denote by $U(k, \omega)$ the Cauchy's matrix for the linear equation

$$
u_{n+1}=A\left(\sigma^{n} \omega\right) u_{k} .
$$

Theorem 5.2. Suppose that the following conditions hold:

(i) there are positive numbers $N$ and $q<1$ such that

$$
\|U(n, \omega)\| \leqslant M q^{n} \quad\left(n \in \mathbb{Z}_{+}\right) ;
$$

(ii) $|F(u, \omega)| \leqslant C+D|u|\left(C \geqslant 0,0 \leqslant D<(1-q) N^{-1}\right)$ for all $u \in E$ and $\omega \in \Omega$.

Then Eq. (21) is dissipative.

Proof. Let $\varphi(\cdot, u, \omega)$ be the solution of Eq. (21) passing through the point $u \in E$ for $n=0$. According to the formula of the variation of constants (see, for example, $[8,9]$ ) we have

$$
\varphi(n, u, \omega)=U(k, \omega) u+\sum_{m=0}^{n-1} U(n-m-1, \omega) F\left(\sigma^{m} \omega, \varphi(m, u, \omega)\right)
$$


and, consequently,

$$
|\varphi(n, u, \omega)| \leqslant N q^{n}|u|+\sum_{m=0}^{n-1} q^{n-m-1}(C+D|\varphi(m, u, \omega)|) .
$$

We set $u(n):=q^{-n}|\varphi(n, u, \omega)|$ and, taking into account (23), obtain

$$
u(n) \leqslant N|u|+C N q^{-1} \sum_{m=0}^{n-1} q^{-m}+D N q^{-1} \sum_{m=0}^{n-1} u(m) .
$$

Denote the right-hand side of inequality (24) by $v(n)$. Note, that

$$
v(n+1)-v(n)=q^{-n} \frac{C N}{q}+\frac{D N}{q} u(n) \leqslant \frac{D N}{q} v(n)+\frac{C N}{q} q^{-n}
$$

and hence,

$$
v(n+1) \leqslant\left(1+\frac{D N}{q}\right) v(n)+\frac{C N}{q} q^{-n} .
$$

From this inequality we obtain

$$
v(n) \leqslant\left(1+\frac{D N}{q}\right)^{n-1} v(1)+\frac{C N}{q} \frac{1-q^{n-1}}{1-q} .
$$

Therefore,

$$
|\varphi(n, u, \omega)| \leqslant(q+D N)^{n-1} q N|u|+\frac{C N}{q-1}\left(q^{n-1}-1\right)
$$

because $v(1)=N|u|$. From (25) follows that

$$
\limsup _{k \rightarrow+\infty}|\varphi(n, u, \omega)| \leqslant \frac{C N}{1-q}
$$

for all $u \in E$ and $\omega \in \Omega$. The theorem is proved.

Let $\left\langle E, \varphi,\left(\Omega, \mathbb{Z}_{+}, \sigma\right)\right\rangle$ be a cocycle over $\left(\Omega, \mathbb{Z}_{+}, \sigma\right)$ with the fiber $E$.

Definition 5.3. A family $\left\{I_{\omega} \mid \omega \in \Omega\right\}\left(I_{\omega} \subset E\right)$ of nonempty compact subsets is called a compact global attractor of the cocycle $\varphi$, if the following conditions are fulfilled:

(i) the set $I:=\bigcup\left\{I_{\omega} \mid \omega \in \Omega\right\}$ is relatively compact;

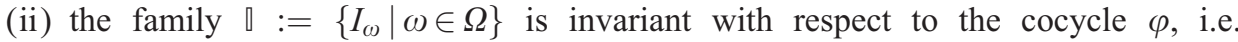
$\varphi\left(n, I_{\omega}, \omega\right)=I_{\sigma^{n} \omega}$ for all $n \in \mathbb{Z}_{+}$and $\omega \in \Omega$;

(iii) the equality

$$
\lim _{n \rightarrow+\infty} \sup _{\omega \in \Omega} \beta(\varphi(n, K, \omega), I)=0
$$

takes place for every $K \in C(E)$, where $C(E)$ is a family of compact sets from $E$. 
Definition 5.4. A cocycle $\langle E, \varphi,(\Omega, \mathbb{T}, \sigma)\rangle$ is said to be dissipative, if there exists a positive number $r$ such that

$$
\limsup _{n \rightarrow+\infty}|\varphi(n, u, \omega)| \leqslant r
$$

for all $u \in E$ and $\omega \in \Omega$.

Theorem 5.5 (Cheban [2,3]). Every dissipative cocycle $\langle E, \varphi,(\Omega, \mathbb{T}, \sigma)\rangle$ admits a compact global attractor $\left\{I_{\omega} \mid \omega \in \Omega\right\}$ such that:

(i) the component $I_{\omega}(\omega \in \Omega)$ is connected;

(ii) the set $I=\bigcup\left\{I_{\omega} \mid \omega \in \Omega\right\}$ is connected, if the space $\Omega$ also is.

Theorem 5.6. Under the conditions of Theorem 5.2, Eq. (21) (i.e. the cocycle $\varphi$ generated by Eq. (21)) admits a compact global attractor $\left\{I_{\omega} \mid \omega \in \Omega\right\}$ with the following properties:

(i) the component $I_{\omega}(\omega \in \Omega)$ is connected;

(ii) the set $I=\bigcup\left\{I_{\omega} \mid \omega \in \Omega\right\}$ is connected, if the space $\Omega$ also is.

Proof. This statement follows directly from Theorems 5.2 and 5.5.

Definition 5.7. A cocycle $\langle E, \varphi,(\Omega, \mathbb{T}, \sigma)\rangle$ is said to be convergent, if it admits a compact global attractor $\left\{I_{\omega} \mid \omega \in \Omega\right\}$ such that every component $I_{\omega}(\omega \in \Omega)$ consists of single point $u_{\omega}$, i.e. $I_{\omega}=\left\{u_{\omega}\right\}(\omega \in \Omega)$.

Theorem 5.8 (Cheban [2,3, Chapter 2]). Let $\langle E, \varphi,(\Omega, \mathbb{T}, \sigma)\rangle$ be a cocycle admitting a compact global attractor $\mathbb{\square}=\left\{I_{\omega} \mid \omega \in \Omega\right\}$. If

$$
\lim _{n \rightarrow+\infty} \max _{\left|u_{1}\right|,\left|u_{2}\right| \leqslant r, \omega \in \Omega}\left|\varphi\left(n, u_{1}, \omega\right)-\varphi\left(n, u_{2}, \omega\right)\right|=0
$$

for every $r>0$, then the set $I_{\omega}$ consists of single point for every $\omega \in \Omega$.

Theorem 5.9. Let $A \in C(\Omega,[E])$ and $F \in C(E \times \Omega, E)$ and the following conditions be fulfilled:

(i) there exist positive numbers $N$ and $q<1$ such that inequality (22) holds;

(ii) $\left|F\left(u_{1}, \omega\right)-F\left(u_{2}, \omega\right)\right| \leqslant L\left|u_{1}-u_{2}\right|\left(0 \leqslant L<N^{-1}(1-q)\right)$ for all $\omega \in \Omega$ and $u_{1}$, $u_{2} \in E$.

Then Eq. (21) is convergent, i.e. the cocycle generated by this equation is convergent.

Proof. (1) We will prove that under the conditions of Theorem 5.9, Eq. (21) admits a compact global attractor $\mathbb{\square}=\left\{I_{\omega} \mid \omega \in \Omega\right\}$. In fact,

$$
|F(u, \omega)| \leqslant|F(0, \omega)|+L|u| \leqslant C+L|u|
$$


for all $u \in E$, where $C:=\max \{|F(0, \omega)| \mid \omega \in \Omega\}$. According to Theorems 5.2 and 5.5, Eq. (21) admits a compact global attractor $\square=\left\{I_{\omega} \mid \omega \in \Omega\right\}$.

(2) Let $\varphi$ be the cocycle generated by Eq. (21). In virtue of the formula of the variation of constants, we have

$$
\varphi(n, u, \omega)=U(n, \omega) u+\sum_{m=0}^{n-1} U(n-m-1, \omega) F\left(\sigma^{m} \omega, \varphi(m, u, \omega)\right) .
$$

Consequently,

$$
\begin{aligned}
\varphi\left(n, u_{1}, \omega\right)-\varphi\left(n, u_{2}, \omega\right)= & U(n, \omega)\left(u_{1}-u_{2}\right) \\
& +\sum_{m=1}^{n-1} U(n-m-1, A)\left[F\left(\sigma^{m} \omega, \varphi(m, u, \omega)\right)\right. \\
& -F\left(\sigma^{m} \omega, \varphi\left(m, u_{2}, \omega\right)\right] .
\end{aligned}
$$

Thus,

$$
\begin{aligned}
\left|\varphi\left(n, u_{1}, \omega\right)-\varphi\left(n, u_{2}, \omega\right)\right| \leqslant & N q^{n}\left(\left|u_{1}-u_{2}\right|\right. \\
& \left.+L q^{-1} \sum_{m=0}^{n-1} q^{-m}\left|\varphi\left(m, u_{1}, \omega\right)-\varphi\left(m, u_{2}, \omega\right)\right|\right) .
\end{aligned}
$$

Let $u(n):=\left|\varphi\left(n, u_{1}, \omega\right)-\varphi\left(n, u_{2}, \omega\right)\right| q^{-n}$. From (27) follows that

$$
u(n) \leqslant N\left(\left|u_{1}-u_{2}\right|+L q^{-1} \sum_{m=0}^{n-1} u(m)\right) .
$$

Denote by $v(n)$ the right-hand side of (28). The following inequality

$$
v(n+1)-v(n)=L N q^{-1} u(n) \leqslant L N q^{-1} v(n)
$$

holds. From (29) we obtain

$$
v(n) \leqslant\left(1+L N q^{-1}\right)^{n-1} v(1)
$$

and since $v(1)=N\left|u_{1}-u_{2}\right|$, we get

$$
u(n) \leqslant\left(1+L N q^{-1}\right) N\left|u_{1}-u_{2}\right| .
$$

From (30) we have

$$
\left|\varphi\left(n, u_{1}, \omega\right)-\varphi\left(n, u_{2}, \omega\right)\right| \leqslant(q+L M)^{n-1} q N\left|u_{1}-u_{2}\right|
$$

for all $u_{1}, u_{2} \in E$ and $\omega \in \Omega$.

To finish the proof of Theorem 5.9 it is sufficient to refer to Theorem 5.8. The theorem is proved.

Remark 5.10. Simple examples show that under the conditions of Theorem 5.6 the compact global attractor $\left\{I_{\omega} \mid \omega \in \Omega\right\}$, generally speaking, is not trivial, i.e. the component set $I_{\omega}$ contains more than one point. This statement can be illustrated by the following example: $u_{n+}=\frac{1}{2} u_{n}+\left(2 u_{n} / 1+u_{n}^{2}\right)$. 
Theorem 5.11. Let $A \in C(\Omega,[E]), F \in C(E \times \Omega, E)$ and the following conditions be fulfilled:

(i) there are positive numbers $N$ and $q<1$ such that

$$
\|U(n, \omega)\| \leqslant N q^{n} \quad(n \geqslant 0, \omega \in \Omega) ;
$$

(ii) $|F(u, \omega)| \leqslant M+\varepsilon|u|(u \in E, \omega \in \Omega)$ and $0 \leqslant \varepsilon \leqslant \varepsilon_{0}<(1-q) /(N(1+q))$;

(iii) the restriction $F_{0}$ of the function $F$ on $\Omega \times B\left[0, r_{0}\right]$, where $r_{0}:=N M(1+q) /$ $(1-q)\left(1-\varepsilon_{0} N(1+q) /(1-q)\right)^{-1}$, satisfies the condition of Lipschitz with the Lipschitz's constant Lip $\left(F_{0}\right)<\frac{1-q}{N(1+q)}$.

Then the following statements hold:

(a) Eq. (21) admits a compact global attractor $\mathbb{\square}=\left\{I_{\omega} \mid \omega \in \Omega\right\}$;

(b) there exists a continuous function $v: \Omega \rightarrow E$ satisfying the following conditions:

(b1) the equality

$$
v(\sigma(n, \omega))=\varphi(n, v(\omega), \omega)
$$

holds for all $n \in \mathbb{Z}_{+}$and $\omega \in \Omega$, where $\varphi(n, u, \omega)$ is the unique solution of Eq. (21) with the initial condition $\varphi(0, u, \omega)=u$.

(b2)

$$
v(\omega) \in I_{\omega} \quad \text { for all } \omega \in \Omega .
$$

Proof. The first statement follows directly from Theorems 5.2 and 5.6.

We will prove now the second affirmation. Define an operator $\mathscr{F}: C\left(\Omega, B\left[0, r_{0}\right]\right) \rightarrow$ $C(\Omega, E)$ in the following way. Let $\alpha \in C\left(\Omega, B\left[0, r_{0}\right]\right)$, then under the conditions of Theorem 5.11 we have $\tilde{f}(\cdot):=F(\cdot, \alpha(\cdot)) \in C\left(\Omega, B\left[0, r_{0}\right]\right)$. According to Theorem 3.7, there exists a unique function $v_{\alpha} \in C(\Omega, E)$ with properties (a) and (b). In virtue of Theorem 3.7, we have

$$
\begin{aligned}
\left\|v_{\alpha}\right\| & \leqslant N \frac{1+q}{1-q} \max _{\omega \in \Omega}|F(\alpha(\omega), \omega)| \\
& \leqslant N \frac{1+q}{1-q}\left(M+\varepsilon_{0}\|\alpha\|\right) \leqslant N \frac{1+q}{1-q}\left(M+\varepsilon_{0} r_{0}\right) \leqslant r_{0}
\end{aligned}
$$

and, consequently, $\mathscr{F}\left(C\left(\Omega, B\left[Q, r_{0}\right]\right)\right) \subseteq C\left(\Omega, B\left[Q, r_{0}\right]\right)$, where $\mathscr{F}: C(\Omega, B[Q, r]) \rightarrow$ $C(\Omega, E)$ is the map defined by the equality $\mathscr{F}(\alpha):=v_{\alpha}$.

Now, we will show that the map $\mathscr{F}: C\left(\Omega, B\left[Q, r_{0}\right]\right) \rightarrow C\left(\Omega, B\left[Q, r_{0}\right]\right)$ is Lipschitzian. In fact, according to Theorem 3.7 we have

$$
\begin{aligned}
\left\|\mathscr{F}\left(\alpha_{1}\right)-\mathscr{F}\left(\alpha_{2}\right)\right\| & \leqslant N \frac{1+q}{1-q} \max _{\omega \in \Omega}\left|F\left(\alpha_{1}(\omega), \omega\right)-F\left(\alpha_{2}(\omega), \omega\right)\right| \\
& \leqslant N \frac{1+q}{1-q} L \max _{\omega \in \Omega}\left|\alpha_{1}(\omega)-\alpha_{2}(\omega)\right| .
\end{aligned}
$$


We note that $N[(1+q) /(1-q)] L \leqslant N[(1+q) /(1-q)] L_{0}<1$. Thus, the map $\mathscr{F}$ is a contraction and, consequently, in virtue of the Banach's fixed point theorem there exists a unique function $w \in C\left(\Omega, B\left[Q, r_{0}\right]\right)$ such that $\mathscr{F}(w)=w$. It is easy to see that $w$ satisfies the first condition. To finish the proof of the theorem it is sufficient to remark that the set $A:=\bigcup\left\{A_{\omega} \mid \omega \in \Omega\right\}$, where $A_{\omega}:=\{w(\omega)\} \times\{\omega\}$, is a compact invariant set in the skew-product dynamical system $\left(X, \mathbb{Z}_{+}, \pi\right)(X:=E \times \Omega$ and $\pi:=(\varphi, \sigma))$ and $J:=\bigcup\left\{J_{\omega} \mid \omega \in \Omega\right\}$ is a maximal compact invariant set of this system. Thus, we have $A \subset J$ and, consequently, $w(\omega) \in I_{\omega}$ for all $\omega \in \Omega$.

Remark 5.12. Under the conditions of Theorem 5.11, dissipativity does not reduce to convergence.

We will give an example that confirms this statement.

Example 5.13. Let $k \in\left(\frac{1}{2}, 1\right)$. Consider a scalar equation

$$
u_{n+1}=k u_{n}+\alpha F\left(u_{n}\right),
$$

where

$$
F(x):= \begin{cases}\frac{x^{2}}{2} & \text { for }|x| \leqslant 10 \\ 50+10[1-\exp (10-|x|)] & \text { for }|x|>10\end{cases}
$$

for $k<1<k+5 \alpha$. We can take $r_{0}:=(2(1-k)) / \alpha$, then it is easy to check that for Eq. (31) all the conditions of Theorem 5.11 are fulfilled for chosen $\alpha, k$, and $F$. In addition, its Levinson's centre (compact global attractor) contains at least two fixed points (in fact, there are 3 of them) and, consequently, Eq. (31) is not convergent.

\section{Almost periodic and recurrent solutions}

Let $\left(X, \mathbb{Z}_{+}, \pi\right)$ be a dynamical system, $x \in X, m \in \mathbb{Z}_{+}, m>0, \varepsilon>0$.

Definition 6.1. A point $x$ is called a stationary point, if $\pi(n, x)=x$ for all $n \in \mathbb{Z}_{+}$. The point $x$ is called $m$-periodic, if $\pi(m, x)=x$.

Definition 6.2. A number $m$ is called an $\varepsilon$-shift ( $\varepsilon$-almost period) of the point $x$, if $\rho(\pi(m, x), x)<\varepsilon\left(\rho(\pi(m+n, x), \pi(n, x))<\varepsilon\right.$ for all $\left.n \in \mathbb{Z}_{+}\right)$.

Definition 6.3. The point $x$ is called almost recurrent (almost periodic), if for any $\varepsilon>0$ there exists a positive number $l \in Z_{+}$such that on every segment (in $\mathbb{Z}_{+}$) of length $l$ there may be found an $\varepsilon$-shift $(\varepsilon$-almost period) of the point $x$.

Definition 6.4. The point $x$ is called recurrent if it is almost recurrent and the set $H(x)=\overline{\left\{\pi(n, x) \mid n \in \mathbb{Z}_{+}\right\}}$is compact.

Denote $\mathfrak{M}_{x}=\left\{\left\{t_{n}\right\} \subset \mathbb{Z}_{+} \mid\left\{\pi\left(t_{n}, x\right)\right\}\right.$ is convergent $\}$. 
Theorem 6.5 (Shcherbakov [15,16]). Let $\left(X, \mathbb{Z}_{+}, \pi\right)$ and $\left(Y, \mathbb{Z}_{+}, \sigma\right)$ be two dynamical systems. Assume that $h: X \rightarrow Y$ is a homomorphism of $\left(X, \mathbb{Z}_{+}, \pi\right)$ onto $\left(Y, \mathbb{Z}_{+}, \sigma\right)$. If a point $x \in X$ is stationary (m-periodic, almost periodic, recurrent), then the point $y:=h(x)$ is also stationary (m-periodic, almost periodic, recurrent) and $\mathfrak{M}_{x} \subset \mathfrak{M}_{y}$.

Definition 6.6. A solution $\varphi(n, u, \omega)$ of Eq. (20) is said to be stationary (m-periodic, almost periodic, recurrent), if the point $x:=(u, \omega) \in X:=E \times \Omega$ is a stationary ( $m$-periodic, almost periodic, recurrent) point of the skew-product dynamical system $\left(X, \mathbb{Z}_{+}, \pi\right)$, where $\pi:=(\varphi, \sigma)$, i.e. $\pi(n,(u, \omega)):=(\varphi(n, u, \omega), \sigma(n, \omega))$ for all $n \in \mathbb{Z}_{+}$ and $(u, \omega) \in E \times \Omega$.

Lemma 6.7. Suppose that $u \in C(\Omega, E)$ satisfies the condition

$$
u(\sigma(n, \omega))=\varphi(n, u(\omega), \omega)
$$

for all $n \in \mathbb{Z}_{+}$and $\omega \in \Omega$. Then the map $h: \Omega \rightarrow X$, defined by

$$
h(\omega):=(u(\omega), \omega)
$$

for all $\omega \in \Omega$, is a homomorphism of $\left(\Omega, \mathbb{Z}_{+}, \sigma\right)$ onto $\left(X, \mathbb{Z}_{+}, \pi\right)$.

Proof. This assertion follows from equalities (32) and (33).

Remark 6.8. A function $u \in C(\Omega, E)$ with property (32) is called a continuous invariant section (or an integral manifold) of nonautonomous difference Eq. (20).

Theorem 6.9. If a function $u \in C(\Omega, E)$ satisfies condition (32) and a point $\omega \in \Omega$ is stationary (m-periodic, almost periodic, recurrent), then the solution $\varphi(n, u(\omega), \omega)$ of Eq. (20) also is stationary (m-periodic, almost periodic, recurrent).

Proof. This statement follows from Theorem 6.5 and Lemma 6.7.

Using Theorem 6.9 and the results from Sections 4 and 6 we obtain some criteria of the existence of periodic (almost periodic, recurrent) solutions of Eq. (15). For example, the following statements hold.

Theorem 6.10. Let $\left(\Omega, \mathbb{Z}_{+}, \pi\right)$ be a dynamical system and $\Omega$ consist of m-periodic (almost periodic, recurrent) points. Then under the conditions of Theorem 4.1, Eq. (15) admits an invariant integral manifold consisting of m-periodic (almost periodic, recurrent) solutions.

Theorem 6.11. Let $\left(\Omega, \mathbb{Z}_{+}, \pi\right)$ be a dynamical system and $\Omega$ consist of m-periodic (almost periodic, recurrent) points. Under the conditions of Theorem 4.2 for a sufficiently small $\lambda$ Eq. (16) admits a single invariant manifold $v_{\lambda}$ consisting of m-periodic (almost periodic, recurrent) solutions of Eq. (16). 
Example 6.12. Consider the equation

$$
u_{n+1}=f\left(n, u_{n}\right)
$$

where $f \in C\left(\mathbb{Z}_{+} \times E, E\right)$; here $C\left(\mathbb{Z}_{+} \times E, E\right)$ is the space of all continuous functions $\mathbb{Z}_{+} \times E \rightarrow E$ ) equipped with a compact-open topology. This topology can be metricised. For example, by the equality

$$
d\left(f_{1}, f_{2}\right):=\sum_{1}^{+\infty} \frac{1}{2^{n}} \frac{d_{n}\left(f_{1}, d_{2}\right)}{1+d_{n}\left(f_{1}, d_{2}\right)},
$$

where $d_{n}\left(f_{1}, d_{2}\right):=\max \left\{\rho\left(f_{1}(k, u), f_{2}(k, u)\right)|k \in[0, n]| u \mid, \leqslant n\right\}$, there is defined a distance on $C\left(\mathbb{Z}_{+} \times E, E\right)$ which generates the topology of pointwise convergence with respect to $n \in \mathbb{Z}_{+}$uniformly with respect to $u$ on every compact from $E$.

Along with Eq. (34), we will consider the $H$-class of Eq. (34)

$$
v_{n+1}=g\left(n, v_{n}\right) \quad(g \in H(f)),
$$

where $H(f)=\overline{\left\{f_{m} \mid m \in \mathbb{Z}_{+}\right\}}$and the over bar denotes the closure in $C\left(\mathbb{Z}_{+} \times E, E\right)$, and $f_{m}(n, u)=f(n+m, u)$ for all $n \in \mathbb{Z}_{+}$and $u \in E$. Denote by $\left(C\left(\mathbb{Z}_{+} \times E, E\right), \mathbb{Z}_{+}, \sigma\right)$ the dynamical system of translations. Here $\sigma(m, g):=g_{m}$ for all $m \in \mathbb{Z}_{+}$and $g \in C\left(\mathbb{Z}_{+} \times\right.$ $E, E)$.

Let $\Omega$ be the hull $H(f)$ of a given function $f \in C\left(\mathbb{Z}_{+} \times E, E\right)$ and denote the restriction of $\left(C\left(\mathbb{Z}_{+} \times E, E\right), \mathbb{R}, \sigma\right)$ on $\Omega$ by $\left(\Omega, \mathbb{Z}_{+}, \sigma\right)$. Let $F: E \times \Omega \rightarrow E$ be a continuous map defined by $F(u, g)=g(0, u)$ for $g \in \Omega$ and $u \in E$. Then Eq. (35) can be rewritten in this form:

$$
u_{n+1}=F\left(u_{n}, \sigma^{n} \omega\right)
$$

where $\omega:=g$ and $\sigma^{n} \omega:=g_{n}$.

Definition 6.13. The function $f \in C\left(\mathbb{Z}_{+} \times E, E\right)$ is said to be periodic (almost periodic, recurrent), if $f \in C\left(\mathbb{Z}_{+} \times E, E\right)$ is a periodic (almost periodic, recurrent) point of the dynamical system of translations $\left(C\left(\mathbb{Z}_{+} \times E, E\right), \mathbb{Z}_{+}, \sigma\right)$.

If the function $f \in C\left(\mathbb{Z}_{+} \times E, E\right)$ is periodic (almost periodic, recurrent), then the set $\Omega:=H(f)$ is the compact minimal set of the dynamical system $\left(C\left(\mathbb{Z}_{+} \times E, E\right), \mathbb{Z}_{+}, \sigma\right)$ consisting of periodic (almost periodic, recurrent) points.

Remark 6.14. Let $\left\langle W, \varphi,\left(\Omega, \mathbb{Z}_{+}, \pi\right)\right\rangle$ be a cocycle over $\left(\Omega, \mathbb{Z}_{+}, \pi\right)$ with fiber $W$ and $w: \Omega \rightarrow W$ be a continuous function satisfying the condition $w(\sigma(n, \omega))=\varphi(n, w(\omega), \omega)$ for all $u \in W$ and $\omega \in \Omega$, then if the point $\omega_{0} \in \Omega$ is stationary (m-periodic, almost periodic, recurrent), then the point $x_{0}:=\left(w\left(\omega_{0}\right), \omega_{0}\right) \in X:=W \times \Omega$ is a stationary ( $m$-periodic, almost periodic, recurrent) point of the triangular map $F: X \rightarrow X(F(u, \omega)$ $:=(\varphi(1, u, \omega), \sigma(1, \omega))$ for all $(u, \omega) \in X)$. 


\section{Pseudo-recurrent solutions}

Definition 7.1 (Cheban et al. [4]). An autonomous dynamical system $\left(\Omega, \mathbb{Z}_{+}, \sigma\right)$ is said to be pseudo-recurrent, if the following conditions are fulfilled:

(a) $\Omega$ is compact;

(b) $\left(\Omega, \mathbb{Z}_{+}, \sigma\right)$ is transitive, i.e. there exists a point $\omega_{0} \in \Omega$ such that $\Omega=\overline{\left\{\sigma^{n} \omega_{0} \mid n \in \mathbb{Z}_{+}\right\}}$;

(c) every point $\omega \in \Omega$ is stable in the sense of Poisson, i.e.

$$
\mathfrak{N}_{\omega}=\left\{\left\{n_{k}\right\} \mid \sigma^{n_{k}} \omega \rightarrow \omega \quad \text { and } \quad n_{k} \rightarrow+\infty\right\} \neq \emptyset .
$$

Lemma 7.2. Let $\left\langle\left(X, \mathbb{Z}_{+}, \pi\right),\left(\Omega, \mathbb{Z}_{+}, \sigma\right), h\right\rangle$ be a nonautonomous dynamical system and the following conditions be fulfilled:

(1) $\left(\Omega, \mathbb{Z}_{+}, \sigma\right)$ is pseudo-recurrent;

(2) $\gamma \in C(\Omega, X)$ is an invariant section of the homomorphism $h: X \rightarrow \Omega$.

Then the autonomous dynamical system $\left(\gamma(\Omega), \mathbb{Z}_{+}, \pi\right)$ is pseudo-recurrent too.

Proof. It is evident that the space $\gamma(\Omega)$ is compact, because $\Omega$ is compact and $\gamma \in C(\Omega, X)$. We note that on the space $\gamma(\Omega)$ we have the dynamical system $(\gamma(\Omega)$, $\left.\mathbb{Z}_{+}, \hat{\pi}\right)$ defined by the homomorphism $\gamma: \Omega \rightarrow \gamma(\Omega)$. Namely, $\hat{\pi}^{n} \gamma(\omega):=\gamma\left(\sigma^{n} \omega\right)$ for all $n \in \mathbb{Z}_{+}$and $\omega \in \Omega$. Hence, $\hat{\pi}^{n} \gamma(\omega)=\pi^{n} \gamma(\omega)$ for all $n \in \mathbb{Z}_{+}$and $\omega \in \Omega$. Now we will show that $\gamma(\Omega)=\overline{\left\{\pi^{n} \gamma\left(\omega_{0}\right) \mid n \in \mathbb{Z}_{+}\right\}}$. Really, let $x \in \gamma(\Omega)$. Then there exists a unique point $\omega \in \Omega$ such that $x=\gamma(\omega)$. Let $\left\{n_{k}\right\} \subset \mathbb{Z}_{+}$be a sequence such that $\sigma^{n_{k}} \omega_{0} \rightarrow \omega$. Then $x=\gamma(\omega)=\lim _{k \rightarrow+\infty} \gamma\left(\sigma^{n_{k}} \omega_{0}\right)=\lim _{k \rightarrow+\infty} \pi^{n_{k}} \gamma(\omega)$ and, consequently, $\gamma(\Omega) \subset$ $\overline{\left\{\pi^{n} \gamma\left(\omega_{0}\right) \mid n \in \mathbb{Z}_{+}\right\}}$. The inverse inclusion is trivial. Thus, $\gamma(\Omega)=\overline{\left\{\pi^{n} \gamma\left(\omega_{0}\right) \mid n \in \mathbb{Z}_{+}\right\}}$. To finish the proof of the lemma it is sufficient to note that $\mathfrak{N}_{\omega} \subseteq \mathfrak{N}_{\gamma(\omega)}$ for every point $\omega \in \Omega$ and, consequently, every point $\gamma(\omega)$ is stable in the sense of Poisson. The lemma is proved.

Lemma 7.2 implies that under the conditions of Theorem 3.7 (respectively, of Theorems 4.1, 5.2 or 5.9) Eq. (32) (respectively, Eqs. (34) or (15)) admits a pseudorecurrent integral manifold.

Therefore, we have the following result.

Theorem 7.3. Assuming that the driving dynamical system $\left(\Omega, \mathbb{Z}_{+}, \sigma\right)$ is pseudorecurrent, and assuming the conditions of Theorem 3.7 (respectively, of Theorems 4.1, 5.2 or 5.9) are satisfied, then Eq. (32) (respectively, Eqs. (34) or (15)) admit a pseudo-recurrent integral manifold.

\section{Chaos in triangular maps}

Let $(X, \rho)$ be a metric space and $\left(X, \mathbb{Z}_{+}, \pi\right)$ be a discrete dynamical system generated by positive powers of the map $f: X \rightarrow X$, i.e. $\pi(n, x)=f^{n} x$ for all $x \in X$ and $n \in \mathbb{Z}_{+}$, where $f^{n}:=f^{n-1} \circ f$. 
Definition 8.1. A subset $M \subseteq X$ is called transitive, if there exists a point $x_{0} \in X$ such that $H\left(x_{0}\right):=\overline{\left\{\pi\left(n, x_{0}\right) \mid n \in \mathbb{Z}_{+}\right\}}=M$.

Definition 8.2. $\{p, q\} \subseteq X$ is called a Li-Yorke pair, if simultaneously

$$
\liminf _{n \rightarrow+\infty} \rho(\pi(n, p), \pi(n, q))=0 \quad \text { and } \quad \limsup _{n \rightarrow+\infty} \rho(\pi(n, p), \pi(n, q))>0 .
$$

Definition 8.3. A set $M \subseteq X$ is called scrambled, if any pair of distinct points $\{p, q\} \subseteq$ $M$ is a Li-Yorke pair.

Definition 8.4. A dynamical system $\left(X, \mathbb{Z}_{+}, \pi\right)$ is said to be chaotic, if $X$ contains an uncountable subset $M$ satisfying the following conditions:

(i) the set $M$ is transitive;

(ii) $M$ is scrambled;

(iii) $\overline{P(M)}=M$, where $P(M):=\left\{x \in M \mid \mathfrak{N}_{x} \neq \emptyset\right\}$ (i.e. $x \in P(M)$, if and only if $x$ is contained in its omega limit set) and by bar we denote the closure in $X$.

Theorem 8.5. Let $\left(X, \mathbb{Z}_{+}, \pi\right)$ and $\left(\Omega, \mathbb{Z}_{+}, \sigma\right)$ be two dynamical systems and $v: X \rightarrow \Omega$ be a homeomorphism of $\left(\Omega, \mathbb{Z}_{+}, \sigma\right)$ onto $\left(X, \mathbb{Z}_{+}, \pi\right)$. Assume that $\left(\Omega, \mathbb{Z}_{+}, \sigma\right)$ is chaotic. Then the dynamical system $\left(X, \mathbb{Z}_{+}, \pi\right)$ is chaotic too.

Proof. Let $\left(\Omega, \mathbb{Z}_{+}, \sigma\right)$ be chaotic and $v: X \rightarrow \Omega$ be a homeomorphism of $\left(\Omega, \mathbb{Z}_{+}, \sigma\right)$ onto $\left(X, \mathbb{Z}_{+}, \pi\right)$. Then there exists a subset $A \subseteq \Omega$ satisfying all the conditions of Definition 8.4. Denote $M:=v(A)$. Then the set $M$ is transitive and $\overline{P(M)}=M$ (see the proof of Lemma 7.2). Note that the set $M:=v(A)$ is uncountable, because the homeomorphism $v: \Omega \rightarrow X$ transforms different points to different points. Finally, to finish the proof of Theorem 8.5 it is sufficient to note that the homeomorphism $v: \Omega \rightarrow$ $X$ maps a $\mathrm{Li}-$ Yorke pair in $\left(\Omega, \mathbb{Z}_{+}, \sigma\right)$ to a Li-Yorke pair in $\left(X, \mathbb{Z}_{+}, \pi\right)$. The theorem is proved.

Remark 8.6. Let $\left\langle W, \varphi,\left(\Omega, \mathbb{Z}_{+}, \pi\right)\right\rangle$ be a cocycle over $\left(\Omega, \mathbb{Z}_{+}, \pi\right)$ with fiber $W$ and $w: \Omega \rightarrow W$ be a continuous function satisfying the condition $w(\sigma(n, \omega))=\varphi(n, w(\omega)$, $\omega)$ for all $u \in W$ and $\omega \in \Omega$, then if the dynamical system $\left(\Omega, \mathbb{Z}_{+}, \pi\right)$ is chaotic the triangular map $F: X \rightarrow X(X:=W$ and $F(u, \omega):=(\varphi(1, u, \omega), \sigma(1, \omega)$ for all $(u, \omega) \in X)$ is chaotic, i.e. the dynamical system $\left(X, \mathbb{Z}_{+}, \pi\right)$ defined by the positive powers of map $F$ is chaotic.

Using Theorem 8.5, Remark 8.6 and the results from Sections 4 and 6 we will obtain some criteria of the existence of chaotic sets for triangular maps. For example the following statements are held.

Theorem 8.7. Let $\left(\Omega, \mathbb{Z}_{+}, \pi\right)$ be a chaotic dynamical system. Then under the conditions of Theorem 4.1, Eq. (15) (respectively, triangular map) admits a compact invariant chaotic set. 


\section{Acknowledgements}

The research described in this publication, in part, was possible due to Award No. MM1-3016 of the Moldovan Research and Development Association (MRDA) and the US Civilian Research \& Development Foundation for the Independent States of the Former Soviet Union (CRDF), and due to the CNR-NATO grant. This paper was written while the first author was visiting University of Macerata (Institute of Economics and Finances) in the winter of 2003. He would like to thank people of that institution for their kind hospitality.

\section{References}

[1] F. Blanchard, E. Glasner, S. Kolyada, A. Mass, On Li-Yorke pairs, J. Reine Angew. Mat. (Crelle's Journal) 547 (2002) 51-68.

[2] D.N. Cheban, Global Attractors of Nonautonomous Dissipative Dynamical Systems, World Scientific, Singapore, 2003, to appear.

[3] D.N. Cheban, Global Attractors of Nonautonomous Dynamical Systems, State University of Moldova, Moldova, 2002 (in Russian).

[4] D.N. Cheban, J. Duan, A. Gherco, Generalization of second Bogoliubov's theorem for non-almost periodic systems, Nonlinear Anal. Real World Appl. 4 (4) (2003) 599-613.

[5] C. Chicone, Yu. Latushkin, Evolution Semigroups in Dynamicals Systems and Differential Equations, American Mathematical Society, Providence, RI, 1999.

[6] I.D. Chueshov, Introduction into the theory of infinite-dimensional dissipative systems, Acta, Kharkiv, 2002.

[7] P. Glendinning, Global attractors of pinched skew-products, Dynamical Systems 17 (23) (2002) $287-294$.

[8] A. Halanay, D. Wexler, Teoria Calitativă a Sistemelor cu Impulsuri, Bucureşti, 1968.

[9] D. Henry, Geometric Theory of Semilinear Parabolic Equations, in: Lecture Notes in Mathematics, Vol. 840, Springer, Berlin, 1981.

[10] P.E. Kloeden, On Sharkovsky’s cycle coexistence ordering, Bull. Austral. Math. Soc. 20 (1979) $171-177$.

[11] S. Kolyada, On dynamics of triangular maps of square, Ergodic Theory Dynamical Systems 12 (1992) 749-768.

[12] C. Robinson, Dynamical Systems, Stability, Symbolic Dynamics and Chaos, CRC Press, Boca Raton Ann Arbor, London, Tokyo, 1995.

[13] G.R. Sell, Topological Dynamics and Ordinary Differential Equations, Van Nostrand, Reinhold, London, 1971.

[14] A.N. Sharkovsky, Yu.L. Maistrenko, E.Yu. Romanenko, Difference Equations and Their Applications, Kluwer Academic Publishers, Dordrecht, Boston, London, 1993.

[15] B.A. Shcherbakov, Topological Dynamics and Poisson's Stability of Solutions of Differential Equations, Kishinev, Shtiintsa, 1972 (in Russian).

[16] B.A. Shcherbakov, Poisson's Stability of Motions of Dynamical Systems and Solutions of Differential Equations, Kishinev, Shtiintsa, 1985 (in Russian). 